\title{
Study of Antigravity in an $F(R)$ Model and in Brans-Dicke Theory with Cosmological Constant
}

\author{
V. K. Oikonomou ${ }^{1}$ and N. Karagiannakis ${ }^{2}$ \\ ${ }^{1}$ Department of Theoretical Physics, Aristotle University of Thessaloniki, 54124 Thessaloniki, Greece \\ ${ }^{2}$ Polytechnic School, Aristotle University of Thessaloniki, 54124 Thessaloniki, Greece \\ Correspondence should be addressed to V. K. Oikonomou; voiko@physics.auth.gr
}

Received 23 May 2014; Revised 23 July 2014; Accepted 24 July 2014; Published 17 August 2014

Academic Editor: Cosimo Bambi

Copyright ( 2014 V. K. Oikonomou and N. Karagiannakis. This is an open access article distributed under the Creative Commons Attribution License, which permits unrestricted use, distribution, and reproduction in any medium, provided the original work is properly cited.

\begin{abstract}
We study antigravity, that is, having an effective gravitational constant with a negative sign, in scalar-tensor theories originating from $F(R)$ theory and in a Brans-Dicke model with cosmological constant. For the $F(R)$ theory case, we obtain the antigravity scalar-tensor theory in the Jordan frame by using a variant of the Lagrange multipliers method and we numerically study the time dependent effective gravitational constant. As we will demonstrate by using a specific $F(R)$ model, although there is no antigravity in the initial model, it might occur or not in the scalar-tensor counterpart, mainly depending on the parameter that characterizes antigravity. Similar results hold true in the Brans-Dicke model.
\end{abstract}

\section{Introduction}

During the last two decades our perception about the universe has changed drastically owing to the discovered late time acceleration that our universe has. Particularly, it can be thought as one of the most striking astrophysical observations with another striking observation being the verification of the inflating period of our universe. Actually, moving from time zero to present time, inflation came first, with the late time acceleration occurring at present epoch. One of the greater challenges in cosmology is to model this late time acceleration in a self-consistent way. According to the new Planck telescope observational data for the present epoch, the universe is consistently described by the $\Lambda$ CDM model, according to which the universe is nearly spatially flat and consists of ordinary matter $(\sim 4.9 \%)$, cold dark matter $(\sim 26.8 \%)$, and dark energy ( 68.3\%). The dark energy is actually responsible for late time acceleration and current research on the field is mostly focused on this issue.

One of the most promising and theoretically appealing descriptions of dark energy and late time acceleration issues is provided by the $F(R)$ modified theories of gravity and related modifications. For important review articles and papers on the vast issue of $F(R)$ theories, the reader is referred to
[1-19] and references therein. For some alternative theories to modified gravity that model dark energy, see [5, 20-24]. The most appealing characteristic of modified gravity theories is that what is actually changed is not the left hand side of the Einstein equations, but the right hand side. Late time acceleration then requires a negative $w$ fluid, which can be consistently incorporated in the energy momentum tensor of these theories. This feature naturally appears in $F(R)$ theories and also late time acceleration solutions of the Friedmann-Robertson-Walker equations naturally occur in these theoretical frameworks $[1-19,25]$. In addition, inflation, the first accelerating period of our universe, is also consistently described by some $F(R)$ theories, rendering the latter a very elegant and economic description of nature at large scales, where general relativity fails to describe phenomena consistently. Particularly, the possibility to theoretically describe, in a consistent and elegant way, earlytime inflation and late-time acceleration in $F(R)$ gravity was explicitly demonstrated in the Nojiri-Odintsov model [25]. For studies on specific solutions in several strong curved backgrounds, see [26-31]. Remarkable possibilities, like modified gravitational theories with nonminimal curvaturematter coupling, were given in [32-35] and references therein. 
In principle, every consistent generalization of general relativity inevitably has to be confronted with the successes of general relativity. Since general relativity is a successful description of nature in strong gravitational environments, there exist a large number of constraints that need to be satisfied, in order that an $F(R)$ modified gravity theory can be considered as viable. The constraints to be satisfied are mainly imposed from local tests of general relativity, for example, from planetary and star formation tests and moreover from various cosmological bounds. In addition, since each $F(R)$ theory has a Jordan frame scalar-tensor gravitational theory counterpart, with $\omega$ zero and a potential, the scalarons of this counterpart theory must be classical, in order to ensure quantum-mechanical stability (see [1-6]).

In theories of modified gravity a longstanding debatable theoretical problem exists, related to Jordan and Einstein frames [36, 37], since the physics coming out from the two frames can be quite different in principle. In view of this, we will focus on the physics of Jordan frame and demonstrate that it is possible to have antigravity [38-41]. For the possibility of antigravity regimes in scalar-tensor theories consult [38-40] and for antigravity in $F(R)$ theories see [41]. In this paper we will study antigravity regimes coming from $F(R)$ theories and from Brans-Dicke theories in the Jordan frame. In reference to the $F(R)$ theories, we will find the Jordan frame antigravity scalar-tensor counterpart, using a modified method of the Lagrange multipliers, as we will see in the following sections. The interesting feature about these theories is that, although the $F(R)$ theory has no antigravity, the resulting Jordan frame scalar-tensor theory may or may not have antigravity. We exemplify this by numerically working out an example. In the case of BransDicke antigravity, we introduce by hand an antigravity term and numerically solve the cosmological equations and as we will demonstrate, similar results hold true; that is, antigravity may exist or not, depending on the parameters of the theory.

This paper is organized as follows: in Section 2 we briefly recall the essentials of $F(R)$ theories; in Section 3 we get to the core of the paper and introduce a modification of the Lagrange multipliers method in order to get antigravity from $F(R)$ theories. Accordingly, we apply the technique to one quite known $F(R)$ model and present the result of our analysis. The study of antigravity is performed in Section 4 and the conclusions follow in the end of the paper.

\section{General Features of $F(R)$ Dark Energy Models in the Jordan Frame}

In this section in order to maintain the paper self-contained, we briefly review the main features of $F(R)$ gravity theories in the Jordan frame in the theoretical framework of the metric formalism. For an important stream of review papers and articles see [1-19] and references therein.

The geometrical background of the manifolds used here is pseudo-Riemannian and is described locally by a Lorentz metric (the FRW metric in our case), in addition to a torsionless, symmetric, and metric compatible affine connection, the so-called Levi-Civita connection. In such a geometric background, the Christoffel symbols are

$$
\Gamma_{\mu \nu}^{k}=\frac{1}{2} g^{k \lambda}\left(\partial_{\mu} g_{\lambda \nu}+\partial_{\nu} g_{\lambda \mu}-\partial_{\lambda} g_{\mu \nu}\right)
$$

and the Ricci scalar becomes

$$
R=g^{\mu \nu}\left(\partial_{\lambda} \Gamma_{\mu \nu}^{\lambda}-\partial_{\nu} \Gamma_{\mu \rho}^{\rho}-\Gamma_{\sigma \nu}^{\sigma} \Gamma_{\mu \lambda}^{\sigma}+\Gamma_{\mu \rho}^{\rho} g^{\mu \nu} \Gamma_{\mu \nu}^{\sigma}\right) .
$$

The $F(R)$ theories of modified gravity are described by a modification of the Einstein-Hilbert action, with the fourdimensional action being equal to

$$
\mathcal{S}=\frac{1}{2 \kappa^{2}} \int d^{4} x \sqrt{-g} F(R)+S_{m}\left(g_{\mu \nu}, \Psi_{m}\right)
$$

where $\kappa^{2}=8 \pi G$ and $S_{m}$ is the matter action containing the matter fields $\Psi_{m}$. For simplicity in this section it will be assumed that the form of the $F(R)$ theory that will be used is $F(R)=R+f(R)$ and in addition the metric formalism framework will be used. Varying the action (3) with respect to the metric $g_{\mu \nu}$, we get the following equations of motion:

$$
\begin{aligned}
& F^{\prime}(R) R_{\mu \nu}(g)-\frac{1}{2} F(R) g_{\mu \nu}-\nabla_{\mu} \nabla_{\nu} F^{\prime}(R) \\
& +g_{\mu \nu} \square F^{\prime}(R)=\kappa^{2} T_{\mu \nu} .
\end{aligned}
$$

In the above equation, $F^{\prime}(R)=\partial F(R) / \partial R$ and also $T_{\mu \nu}$ is the energy momentum tensor.

The most striking feature of the $F(R)$ modified gravity theories is that what actually changes in reference to the usual Einstein-Hilbert gravity equations is the right hand side of the Einstein equations and not the left, which remains the same. Indeed, the equations of motion (4) can be cast in the following form:

$$
\begin{aligned}
R_{\mu \nu}-\frac{1}{2} R g_{\mu \nu} & \\
=\frac{\kappa^{2}}{F^{\prime}(R)}\left(T_{\mu \nu}+\frac{1}{\kappa}\right. & {\left[\frac{F(R)-R F^{\prime}(R)}{2} g_{\mu \nu}+\nabla_{\mu} \nabla_{\nu} F^{\prime}(R)\right.} \\
& \left.\left.\quad-g_{\mu \nu} \square F^{\prime}(R)\right]\right) .
\end{aligned}
$$

Therefore we get an additional contribution for the energy momentum tensor, coming from the term

$$
\begin{aligned}
T_{\mu \nu}^{\mathrm{eff}}=\frac{1}{\kappa}[ & \frac{F(R)-R F^{\prime}(R)}{2} g_{\mu \nu} \\
& \left.+\nabla_{\mu} \nabla_{\nu} F^{\prime}(R)-g_{\mu \nu} \square F^{\prime}(R)\right] .
\end{aligned}
$$

It is this term that actually models the dark energy in $F(R)$ theories of modified gravity. Taking the trace of (4) we straightforwardly obtain the following equation:

$$
3 \square F^{\prime}(R)+R F^{\prime}(R)-2 F(R)=\kappa^{2} T,
$$


where $T$ stands for the trace of the energy momentum tensor $T=g^{\mu \nu} T_{\mu \nu}=-\rho+3 P$ and, additionally, $\rho$ and $P$ stand for the matter energy density and pressure, respectively.

There exists another degree of freedom in $F(R)$ theories, as can be easily seen by observing (7). This degree of freedom is actually a scalar degree of freedom, called scalaron, described by the function $F^{\prime}(R)$, with (7) being the equation of motion of this scalar field. In a flat Friedmann-LemaitreRobertson-Walker spacetime, the Ricci scalar is equal to

$$
R=6\left(2 H^{2}+\dot{H}\right)
$$

with $H$ being the Hubble parameter and the "dot" indicating differentiation with respect to time. The cosmological equations of motion are given by the following set of equations:

$$
\begin{aligned}
3 F^{\prime}(R) H^{2}= & \kappa^{2}\left(\rho_{m}+\rho_{r}\right) \\
& +\frac{\left(F^{\prime}(R) R-F(R)\right)}{2}-3 H \dot{F}^{\prime}(R), \\
-2 F^{\prime}(R) \dot{H}= & \kappa^{2}\left(p_{m}+\frac{4}{3} \rho_{r}\right)+F \ddot{F}^{\prime}(R)-H \dot{F}^{\prime}(R),
\end{aligned}
$$

with $\rho_{r}$ and $\rho_{m}$ standing for the radiation and matter energy density, respectively. Thereby, the total effective energy density and pressure of matter and geometry are [1-6]

$$
\begin{gathered}
\rho_{\mathrm{eff}}=\frac{1}{F^{\prime}(R)}\left[\rho_{m}+\frac{1}{\kappa^{2}}\left(F^{\prime}(R) R-F(R)-6 H \dot{F}^{\prime}(R)\right)\right], \\
p_{\mathrm{eff}}=\frac{1}{F^{\prime}(R)}\left[p_{m}+\frac{1}{\kappa^{2}}\left(-F^{\prime}(R) R+F(R)\right.\right. \\
\left.\left.+4 H \dot{F}^{\prime}(R)+2 \ddot{F}^{\prime}(R)\right)\right]
\end{gathered}
$$

where $\rho_{m}, P_{m}$ denote the total matter energy density and matter pressure, respectively.

\section{Antigravity in $F(R)$ Models}

The possibility of antigravity sectors in $F(R)$ theories was firstly pointed out in [41] and also in various scalar-tensor models in [38-40]. In most cases, a passing from antigravity to a gravity regime always occurs, with a singularity existing at the transition between these two different gravitational regimes. At the transition, the effective gravitational constant and also several invariants of the geometry, such as the Weyl invariant, become singular quantities [38-41]. In the present paper, we are interested in studying the time dependence of the effective gravitational constant and see how this behaves for both an $F(R)$ theory related antigravity scalar-tensor model and an antigravity version of the Brans-Dicke model with cosmological constant. In reference to $F(R)$ theories, we will explicitly demonstrate in the next subsection how to find the antigravity scalar-tensor theory in the Jordan frame. By doing so, we will have at hand an antigravity scalartensor theory with a potential term and we will explicitly find how the scalar field, along with the gravitational constant and the energy density, behaves for various values of the model dependent and cosmological variables. Then we study the Brans-Dicke model in which we will make a by hand modification in order to render it an antigravity model. As we will see, in both cases, there exist several gravityantigravity regimes, depending on the values of the model dependent and cosmological variables. Moreover, for the $F(R)$ model, although the model per se has no antigravity, the corresponding scalar-tensor model gives rise to antigravity regimes. However, there exist values of the variables for which the model describes gravity regimes. In the following subsections we will study in detail these models.

3.1. A General Way to Obtain Antigravity Scalar-Tensor Models from $F(R)$ Models. It is a quite well-known fact that scalar-tensor theories are equivalent to $F(R)$ theories. In the literature one starts from an $F(R)$ theory and ends up to a nonminimally coupled scalar-tensor theory and more specifically to a Brans-Dicke theory with $\omega_{\mathrm{BD}}$ equal to zero. This is practically the Lagrange multipliers method (see [1-6] and particularly Nojiri and Odintsov [1] and De Felice and Tsujikawa [2]).

In this paper we will use a variant but quite similar method to obtain an antigravity scalar-tensor theory starting from a given $F(R)$ theory. Consider the general $F(R)$ theory with matter, which is described by the action

$$
\mathcal{S}=\int d^{4} x \sqrt{-g} F(R)+S_{m}\left(g_{\mu \nu}, \Psi_{m}\right) .
$$

Introducing an auxiliary field $\chi$, which acts as a Lagrange multiplier, the action (11) becomes

$$
\begin{aligned}
\mathcal{S}=\int & d^{4} x \sqrt{-g}\left(F(\chi)+F_{, \chi}(\chi)(R-\chi)\right) \\
& +S_{m}\left(g_{\mu \nu}, \Psi_{m}\right)
\end{aligned}
$$

with $F_{, \chi}(\chi)$ being the first derivative of the function $F(\chi)$ with respect to $\chi$. By varying the action (12) with respect to $\chi$ we obtain

$$
F_{, \chi \chi}(\chi)(R-\chi)=0 .
$$

Given that $F_{, \chi \chi}(\chi) \neq 0$, which is actually true for most viable $F(R)$ theories, we may conclude that $R=\chi$. Hence, the action (12) actually recovers the initial $F(R)$ gravity action (11). We define

$$
\varphi-\mathscr{B}=F_{, \chi}(\chi)
$$

and the action of (12) is expressed as a function of the field $\varphi$ in the following way:

$$
\mathcal{S}=\int d^{4} x \sqrt{-g}[(\varphi-\mathscr{B}) R-U(\varphi)]+S_{m}\left(g_{\mu \nu}, \Psi_{m}\right) .
$$

Comparing the nonminimal coupling term $(\varphi-\mathscr{B}) R$ to the corresponding term $(1 / 16 \pi G) R$ of the standard EinsteinHilbert action, we get the relation for the effective gravitational constant

$$
G_{\mathrm{eff}}=\frac{1}{16 \pi(\varphi-\mathscr{B})} .
$$


It is easy to see that if $\varphi(t)-\mathscr{B}=F_{, \chi}(\chi)<0$ there emerges antigravity. The potential term $U(\varphi)$ is equal to

$$
U(\varphi)=\chi(\varphi)(\varphi-\mathscr{B})-F(\chi(\varphi)),
$$

where the function $\chi(\varphi)$ is directly obtained by solving the algebraic equation (14) with respect to $\chi$, so that $\chi$ is an explicit function of $\varphi$. Therefore as result, starting from an $F(R)$ theory and using the technique we just presented, one obtains Jordan frame antigravity scalar-tensor theories.

3.2. The Model $F(R)=R-R^{-p}$ with $p$ a Positive Integer. As an application of the method we just presented, let us use a viable $F(R)$ model a modified version of which is quite frequently used in $F(R)$ cosmology [1-6]. The model has the following form as a function of the curvature scalar $R$ :

$$
F(R)=R-R^{-p},
$$

with $p$ being some positive integer number. This form of the $F(R)$ function ensures that the first derivative of the $F(R)$ function with respect to $R$ is positive definite for $R \geq R_{D}$, with $R_{D}$ being the final de-Sitter attractor solution of the theory; that is,

$$
\frac{d F(R)}{d R}>0
$$

Condition (19) assures that no antigravity occurs for the $F(R)$ model [1-6]. However, as we will demonstrate, antigravity might occur in the Jordan frame scalar-tensor model. The action corresponding to the $F(R)$ action (18) is the following:

$$
\mathcal{S}=\int d^{4} x \sqrt{-g}\left(R-R^{-p}\right)+S_{m}\left(g_{\mu \nu}, \Psi_{m}\right) .
$$

Using the Lagrange multipliers method we introduced in the previous section, we obtain the corresponding scalar-tensor antigravity theory, with the Jordan frame action being equal to

$$
\mathcal{S}=\int d^{4} x \sqrt{-g}\left[(\varphi-\mathscr{B}) R-U_{F(R)}(\varphi)\right]+S_{m}\left(g_{\mu \nu}, \Psi_{m}\right)
$$

The potential $U_{F(R)}(\varphi)$ for the present $F(R)$ model is equal to

$$
\begin{aligned}
U_{F(R)}(\varphi)= & \left(\frac{p}{\varphi-\mathscr{B}-1}\right)^{1 /(p+1)}(\varphi-\mathscr{B}) \\
& -\left(\frac{p}{\varphi-\mathscr{B}-1}\right)^{1 /(p+1)} \\
& +\left(\frac{p}{\varphi-\mathscr{B}-1}\right)^{-(p /(p+1))} .
\end{aligned}
$$

Having action (21) at hand, along with potential term (22), we can study the antigravity scalar-tensor model in a straightforward way. By varying action (21) with respect to the metric and the scalar field, we get the Einstein equations that describe the cosmic evolution of the antigravity $F(R)$-related scalar-tensor model. Assuming a flat FRW metric of the form

$$
d s^{2}=-d t^{2}+a^{2}(t) \sum_{i} d x_{i}^{2}
$$

the cosmological equations are equal to

$$
\begin{gathered}
3(\varphi-\mathscr{B}) H^{2}=\rho+U_{F(R)}(\varphi)-3 H \dot{\varphi}, \\
-2(\varphi-\mathscr{B}) \dot{H}=\rho+P+\ddot{\varphi}-H \dot{\varphi}, \\
-R+2 \frac{d U_{F(R)}(\varphi)}{d \varphi}=0
\end{gathered}
$$

where $\dot{\varphi}$ denotes differentiation of the scalar field function $\varphi(t)$, with respect to the time variable $t$ :

$$
\dot{\varphi}=\frac{d \varphi}{d t} .
$$

In addition $P=w \rho$ and also the continuity equation for matter, stemming from $T_{; \mu}^{\mu \nu}=0$, holds true:

$$
\dot{\rho}+3 H(1+w) \rho=0 .
$$

From (21), it easily follows that the effective gravitational constant of the Jordan frame scalar-tensor theory is equal to

$$
G_{\text {eff }}(t)=\frac{1}{16 \pi(\varphi(t)-\mathscr{B})} .
$$

We numerically solved the cosmological equations (24a), (24b), and (24c) and in Figures 1 and 2 we present the results which we will now analyze in detail. As a general comment let us note that, depending on the value of the antigravity parameter $\mathscr{B}$, the Jordan frame scalar-tensor theory may or may not have antigravity. Therefore, although we started with an $F(R)$ theory with no antigravity solutions, the Jordan frame counterpart exhibits antigravity for some values of the parameter $\mathscr{B}$. In order for the time dependent functions $\varphi, \rho$, and $G_{\text {eff }}$ to vary smoothly, we chose the initial conditions to be

$$
\rho(1)=1, \quad \varphi(1)=1, \quad \dot{\varphi}(1)=0, \quad t \times H(1) \sim 1,
$$

which are similar to those used in [42] (check also [37]). We also performed the following rescaling for time:

$$
t=1 \longrightarrow 10^{-46} \mathrm{sec}
$$

in favor of the simplicity of the plots. The above initial conditions and time scaling are used for all the plots in this paper. The results obtained by the numerical analysis are qualitatively robust towards the change of the initial conditions, meaning that the only thing that changes is not the whole phenomenon, but the exact time point when the singularity occurs; in all cases the transition singularity occurs long before the beginning of inflation at $t=10^{10} \rightarrow$ $10^{-36} \mathrm{sec}$. In Figure 1 we provide plots of the scalar field 


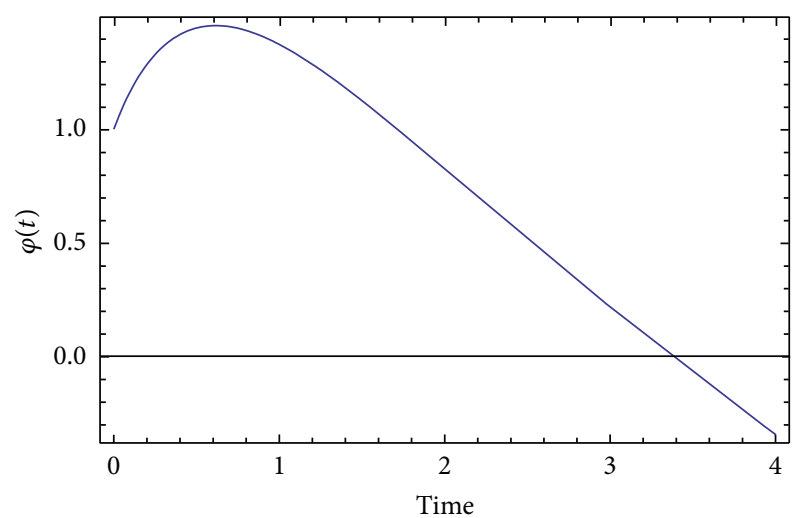

(a)

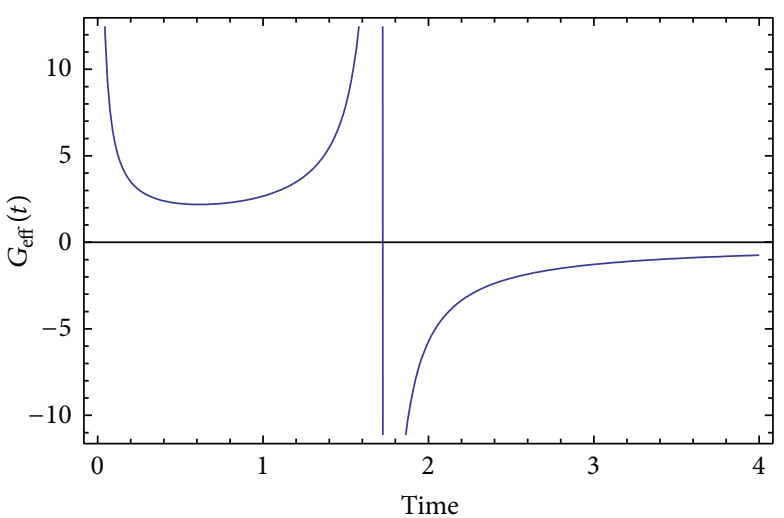

(b)

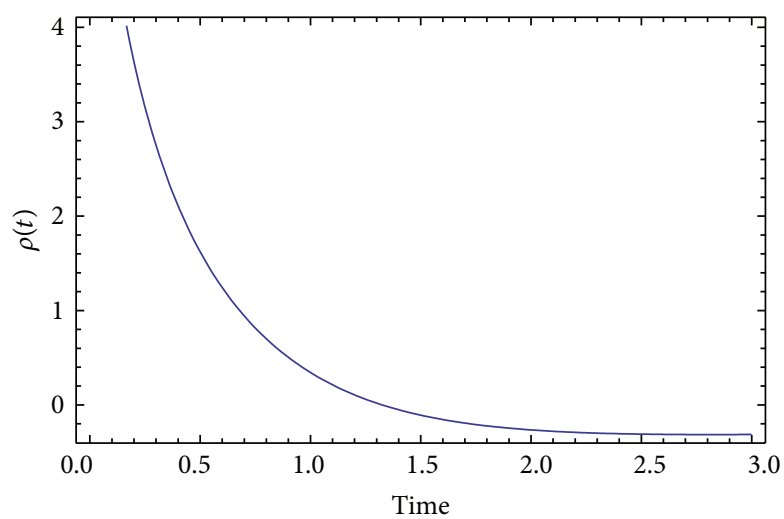

(c)

FIGURE 1: $F(R)$ model: time dependence of the scalar field $\varphi(t)$ (a), the effective gravitational constant $G_{\text {eff }}(t)(b)$, and the matter energy density $\rho(t)(\mathrm{c})$, for $w=1 / 3, p=3$, and $\mathscr{B}=1$.

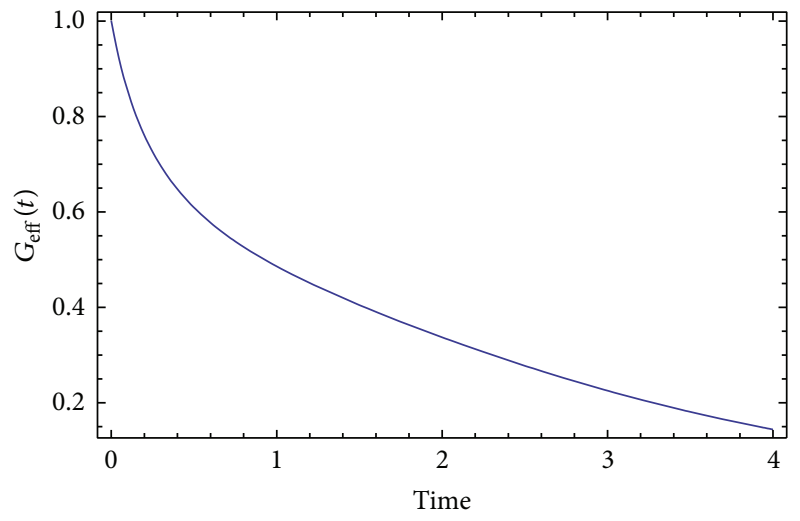

(a)

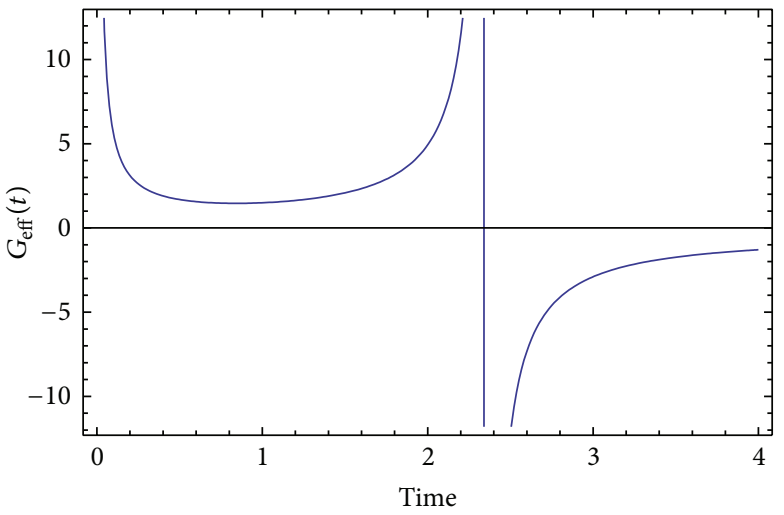

(b)

FIGURE 2: $F(R)$ model: the effective gravitational constant $G_{\text {eff }}(t)$ as a function of time, for nonrelativistic matter $w=0$, with $\mathscr{B}=0.001$, $p=2($ a) and $\mathscr{B}=1, p=2($ b) .

$\varphi(t)$, the energy density $\rho(t)$, and the effective gravitational constant $G_{\text {eff }}(t)$ as a function of the time $t$, with the time axis properly rescaled. We have chosen the numerical values to be $w=1 / 3, p=3$, and $\mathscr{B}=1$, that is, in a radiation dominated universe. The same behavior however is observed for $\mathscr{B}=1$ and different values for $w$. Therefore, we observe that the parameter $\mathscr{B}$ critically affects the antigravity behavior. In the present case, the occurring antigravity can be seen in the right part of Figure 1; as can be seen, there appears a gravity dominated period for $0<t<1.7$ and after the singularity at $t=1.7$ antigravity occurs. In Figure 2, we present the time dependence of the effective gravitational constant $G_{\text {eff }}(t)$, for 
two different values of $\mathscr{B}$, namely, $\mathscr{B}=0.001$ (a) and $\mathscr{B}=1$ (b). We assumed a universe filled with nonrelativistic matter, that is, $w=0$ and also $p=2$. As we can see in this case, for $\mathscr{B}=0.001$ there is no antigravity and conversely for $\mathscr{B}=1$ there is. This is the expected behavior of the Jordan frame theory, since as $\mathscr{B}$ increases, the possibility that the term $(\varphi(t)-\mathscr{B})$ becomes negative increases, depending, of course, on the initial conditions and on the other parameters' values.

The model we studied in this section is similar to the one studied in [41], in which case the antigravity scalar-tensor model was the following:

$$
S=\int d x^{4} \sqrt{-g}\left[\frac{1-\varphi^{2}}{12} R-\frac{1}{2} g^{\mu \nu} \partial_{\mu} \varphi \partial_{\nu} \varphi-J(\varphi)\right] .
$$

The corresponding $F(R)$ gravity action, following the technique presented in [41] is easily found to be

$$
S=\int d x^{4} \sqrt{-g} F(R)
$$

where $F(R)$ stands for

$$
F(R)=\frac{e^{\eta(\varphi(R))}}{12}\left(1-\varphi^{2}(R)\right) R-e^{2 \eta(\varphi(R))} J(\varphi(R)) .
$$

Moreover, the real function $\eta(\varphi)$ satisfies

$$
\left(1+2 \varphi^{2}\right) \eta^{\prime}(\varphi)^{2}-4 \eta^{\prime}(\varphi)-4=0
$$

and, as a result, the kinetic term of the scalar field vanishes. This antigravity model clearly provides us with regimes governed by a negative gravitational constant for some values of the scalar field $\varphi$, clearly indicating a highly nonsmooth, big crunch-big bang transition in the theoretical context of [41].

Before we close this section, we discuss an important issue. Reasonably, it can be argued that since the effective gravitational constant $G_{\text {eff }}(t)$ diverges at some time, this could imply some sort of instability of the $F(R)$ theory. Indeed, this is true to some extent. Actually, the singularity of the gravitational constant is a spacetime one, since spacetime geometric invariants like the Kretschmann scalar $R_{a b c d} R^{a b c d}$ seriously diverge. In a mathematical context, this singularity is also a naked Cauchy horizon, not "dressed" by some event horizon, which, in turn, would imply the loss of predictability and also signal a spacetime singularity. Therefore, it is better if these singularities occur in the very early universe. As for the issue of stability of the initial $F(R)$ theory, this is an involved question, since the quantum mechanical stability of the $F(R)$ theory is examined in the Einstein frame and not in the Jordan frame [1]. In the case of an occurring singularity, the Einstein frame is not consistently defined, since this singularity also introduces another singularity in the scalar field redefinition necessary for the definition of the canonical transformation in the Einstein frame (see the book of Faraoni for more details on this [36]). A very thorough analysis of the stability of a, similar to ours, scalar-tensor model was studied in [43] (see equation (1) of [43]), in which case the model can exhibit antigravity if the nonminimal coupling term becomes negative. The model in [43] can be identical to our Brans-Dicke model if the potential is zero and the nonminimal coupling contains terms of the order of $\sim \varphi$.

\section{Antigravity in Brans-Dicke Models}

As we saw in the previous section, even though we started from an $F(R)$ theory with no antigravity, the antigravity Jordan frame action may or may not have antigravity solutions. In this section, we will study a minor modification of the Brans-Dicke model with cosmological constant. The antigravity term will be introduced by hand and will be of the form $(\varphi-\mathscr{B}) R$, with $\mathscr{B}$ being the extra term introduced by hand. The general action in the Jordan frame that describes a general Brans-Dicke model with cosmological constant, potential $U(\varphi)$, and matter is

$$
\begin{aligned}
S= & \int d x^{4} \sqrt{-g}\left[\frac{1}{2} \varphi(R-2 \Lambda)-\frac{\omega_{\mathrm{BD}}}{\varphi} g^{\mu \nu} \partial_{\mu} \varphi \partial_{\nu} \varphi-U(\varphi)\right] \\
& +\int d x^{4} \sqrt{-g} L_{\text {matter }} .
\end{aligned}
$$

In the following, we will assume that, initially, the scalar potential $U(\varphi)$ is zero and also that the cosmological constant is positive and has the value $\Lambda=10^{-49} \mathrm{GeV}^{4}$. The antigravity model we will study is obtained from the original BrandDicke model with cosmological constant (34) if we modify by hand the action in the following way:

$$
\begin{aligned}
S= & \int d x^{4} \sqrt{-g}\left[\frac{1}{2}(\varphi-\mathscr{B}) R-\frac{\omega_{\mathrm{BD}}}{\varphi} g^{\mu \nu} \partial_{\mu} \varphi \partial_{\nu} \varphi-\varphi \lambda\right] \\
& +\int d x^{4} \sqrt{-g} L_{\text {matter }} .
\end{aligned}
$$

The term $\varphi \lambda$ acts as a potential term and hence we have at hand an antigravity Brans-Dicke model with potential $U_{\mathrm{BD}}(\varphi)=\varphi \Lambda$. By varying (35) with respect to the metric and the scalar field, we obtain the Einstein equations describing the cosmological evolution of the antigravity Brans-Dicke model, which for a flat FRW metric are equal to

$$
\begin{gathered}
3(\varphi-\mathscr{B}) H^{2}=\rho^{(m)}+\frac{\omega_{\mathrm{BD}}}{2}(\dot{\varphi})^{2}+\varphi \Lambda-3 H \dot{\varphi}, \\
-2(\varphi-\mathscr{B}) \dot{H}=\rho^{(m)}+P^{(m)}+\omega_{\mathrm{BD}}(\dot{\varphi})^{2}+\ddot{\varphi}-H \dot{\varphi}, \\
\ddot{\varphi}+3 H \dot{\varphi}+\frac{1}{2 \omega_{\mathrm{BD}}}[-R+2 \Lambda]=0 .
\end{gathered}
$$

In the following, we will take $\omega_{\mathrm{BD}}=1 / 2$. As in the previous case, the effective gravitational constant varies with time in the Jordan frame model and its value is given by

$$
G_{\text {eff }}(t)=\frac{1}{16 \pi(\varphi(t)-\mathscr{B})} .
$$

We have solved numerically the cosmological equations (36a), (36b), and (36c) and as a general remark let us note 


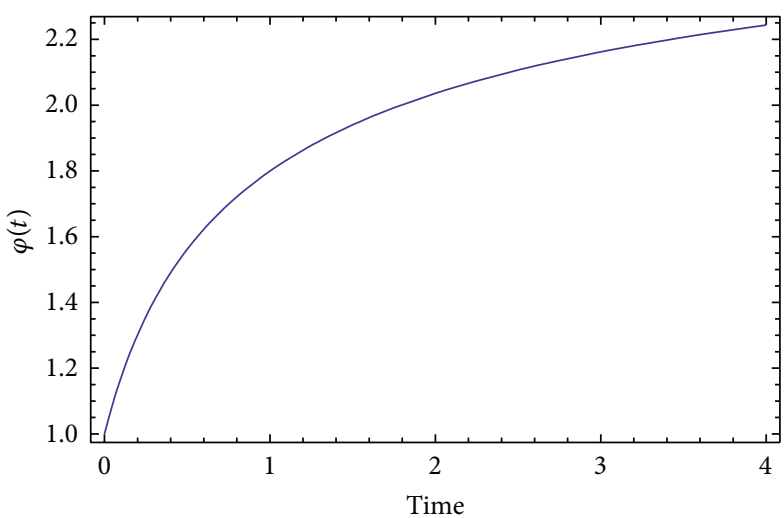

(a)

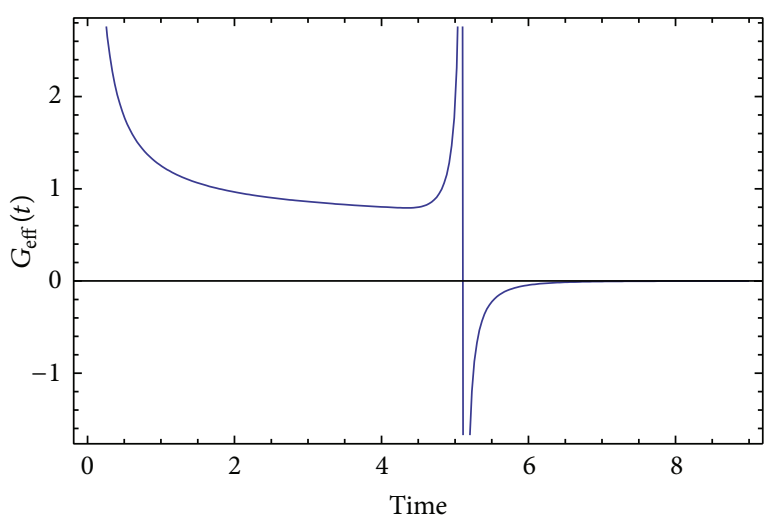

(b)

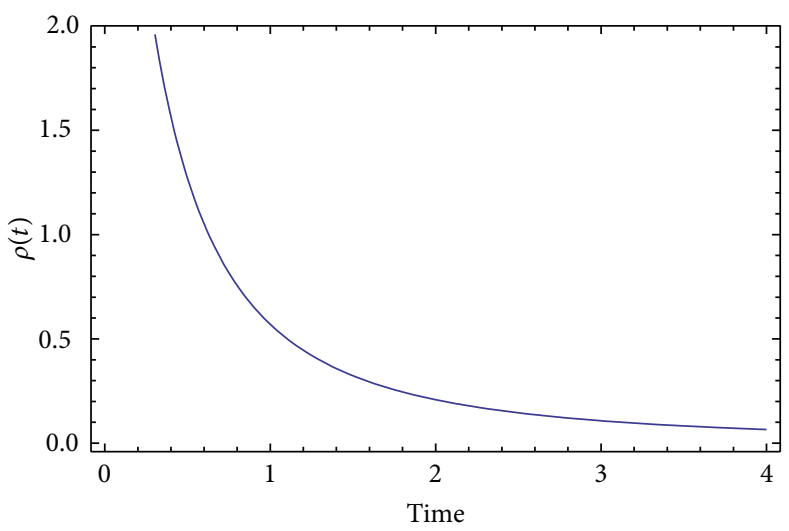

(c)

FIGURE 3: Brans-Dicke model with cosmological constant: time dependence of the scalar field $\varphi(t)$ (a), the effective gravitational constant $G_{\text {eff }}(t)(\mathrm{b})$, and the matter energy density $\rho(t)(\mathrm{c})$, for $w=1 / 3, \mathscr{B}=1$, and the cosmological constant $\Lambda=10^{-49}$.

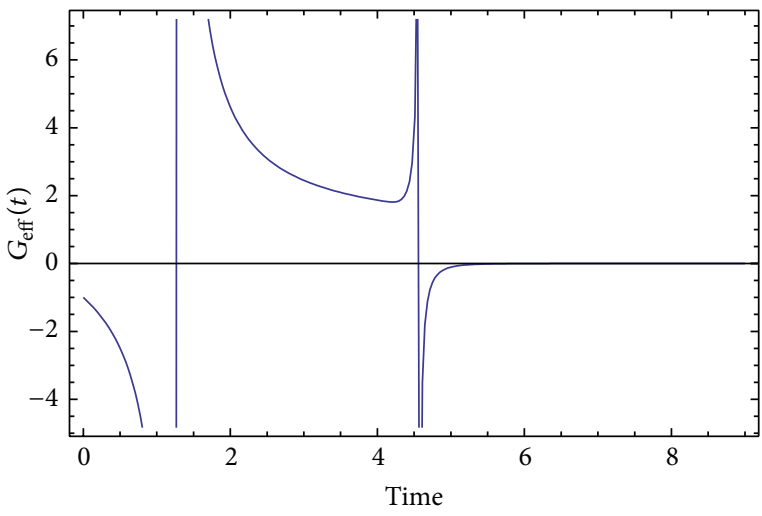

(a)

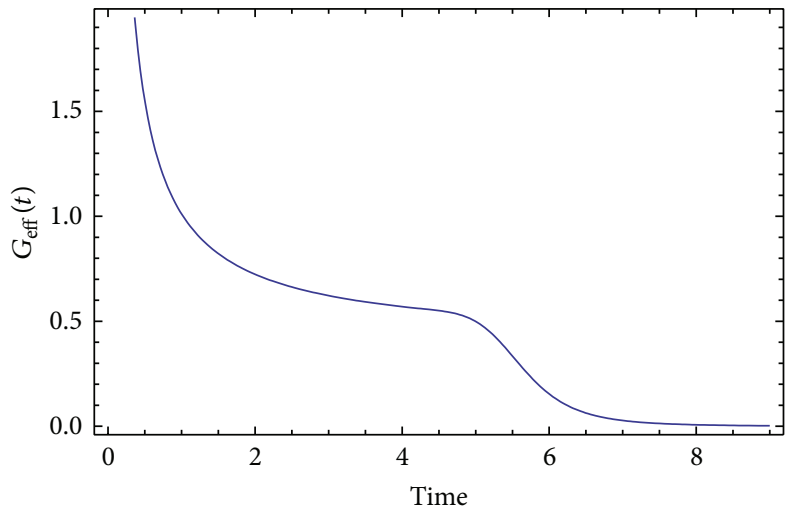

(b)

FIGURE 4: Brans-Dicke model with cosmological constant: the effective gravitational constant $G_{\text {eff }}(t)$ as a function of time, for nonrelativistic matter $w=0$, with $\Lambda=10^{-49}$, and $\mathscr{B}=2$ (a) and $\mathscr{B}=1$ (b).

that the model has both gravity and antigravity solutions, depending on the values of the parameters and specifically on the value of the antigravity parameter $\mathscr{B}$. In Figures 3 and 4 we have presented the results of our numerical analysis for various parameter values and we now discuss them in detail. In Figure 1 appears the time dependence of the scalar field $\varphi(t)$, the energy density $\rho(t)$, and the effective gravitational constant $G_{\text {eff }}(t)$, where again we have properly rescaled the time axis. The numerical values we used in Figure 3 are $w=$ $1 / 3, \mathscr{B}=1$, and $\Lambda=10^{-49}$. Changing the value of $w$ does not drastically affect the solutions, which crucially depend on the value of the antigravity parameter $\mathscr{B}$. As can be seen from 
the time dependence of the effective gravitational constant $G_{\text {eff }}(t)$ in Figure 3, antigravity occurs along with a singularity between the transition from gravity to antigravity. This latter feature is quite common in antigravity models (see, e.g., [3841]). Accordingly, in Figure 4 we have provided the plots of the effective gravitational constant as a function of time, for $w=0, \Lambda=10^{-49}$, and $\mathscr{B}=2$ (1) for (a) and (b) plots. Obviously, for $\mathscr{B}=2$ (a) a complex antigravity pattern occurs, while for $\mathscr{B}=1$ (b) there is no antigravity at all. This result validates our observation that antigravity crucially depends on the values of the $\mathscr{B}$.

\section{A Brief Discussion}

Before closing this section, we discuss a last issue of some importance. It is generally known that a general $F(R)$ theory with the method of Lagrange multipliers can be transformed to a Brans-Dicke theory with $\omega_{\mathrm{BD}}=0$ and nonzero potential. Indeed, it is easy to see this and we demonstrate it shortly. Consider a general $F(R)$ theory described by the following action:

$$
\mathcal{S}=\int d^{4} x \sqrt{-g} F(R)+S_{m}\left(g_{\mu \nu}, \Psi_{m}\right)
$$

We introduce an auxiliary field $\chi$, which actually is the Lagrange multiplier. Using this field, the action (38) becomes

$$
\mathcal{S}=\int d^{4} x \sqrt{-g}\left(F(\chi)+F_{, \chi}(\chi)(R-\chi)\right)+S_{m}\left(g_{\mu \nu}, \Psi_{m}\right),
$$

with $F_{, \chi}(\chi)$ being the first derivative of the function $F(\chi)$ with respect to $\chi$. Varying the action (39), with respect to the auxiliary field $\chi$, we get

$$
F_{, \chi \chi}(\chi)(R-\chi)=0 .
$$

Recalling that $F_{, \chi \chi}(\chi) \neq 0$, which actually holds true for most viable $F(R)$ theories, we get $R=\chi$. Therefore, the action (39) recovers the initial $F(R)$ gravity action (39). If we define

$$
\varphi=F_{, \chi}(\chi)
$$

then the action appearing in (39) becomes actually a function of the field $\varphi$, as can be seen below:

$$
\mathcal{S}=\int d^{4} x \sqrt{-g}[\varphi R-U(\varphi)]+S_{m}\left(g_{\mu \nu}, \Psi_{m}\right) .
$$

The scalar potential term $U(\varphi)$ is equal to the following expression:

$$
U(\varphi)=\chi(\varphi) \varphi-F(\chi(\varphi))
$$

Solving the algebraic equation (41) with respect to $\chi$ will actually give us in closed form the function $\chi(\varphi)$ (at least in most cases), as a function of $\varphi$. Therefore, it is a straightforward way to obtain a Brans-Dicke theory with $\omega_{\mathrm{BD}}$ zero and nonzero potential by starting from a general $F(R)$ theory. A question naturally springs to mind, that is, whether it is possible to have any sort of coincidence between $F(R)$ gravity and BransDicke with a nonzero potential and zero $\omega_{\mathrm{BD}}$ and the answer is actually yes, but only when the potential of the Brans-Dicke is exactly the one of (43). Now, one has to be cautious, however, because this coincidence is "one way" only, meaning that if we start with the Brans-Dicke theory with $\omega_{\mathrm{BD}}=0$ and we try to find the corresponding $F(R)$ theory by using a conformal transformation, then we may end up with a different $F(R)$ theory, which we denote, for example, $f(R)$. This requires a much deeper study that extends beyond the purpose of this paper and we defer this interesting issue to a near future work. However, the reader is referred to the method in four dimensions used by the authors in [41]. There, it can be seen that, when starting from a general scalar-tensor theory, we end up with a certain class of $F(R)$ theories determined by a constraint which the scalar field has to obey. It is not obvious, however, that starting from a Brans-Dicke theory with $\omega_{\mathrm{BD}}=$ 0 and nonzero potential, we will end up to the original $F(R)$ theory we started with. We hope to answer this issue in a future paper.

\section{Conclusions}

In this paper we studied antigravity in scalar-tensor theories originating from $F(R)$ theories and also antigravity in the Brans-Dicke model with cosmological constant. In the case of the $F(R)$ theories we used a variant of the Lagrange multipliers method leading to antigravity scalar-tensor model in the Jordan frame, with $\omega=0$ and a scalar potential. We applied the technique and studied numerically the timedependence of the gravitational constant. As we exemplified, although the initial $F(R)$ model has no antigravity, guaranteed by the condition $F^{\prime}(R)>0$, the scalar-tensor Jordan frame counterpart may or may not have antigravity. This latter feature strongly depends on the parameters of the theory and particularly on the antigravity parameter $\mathscr{B}$. In the case of the Brans-Dicke model with cosmological constant, we studied a by-hand introduced antigravity modification of the model in the Jordan frame. The numerical analysis of the cosmological equations showed that the model exhibits antigravity depending on the numerical values of the parameters and particularly on the $\mathscr{B}$ antigravity parameter, like in the $F(R)$ model case. In both cases, there exist regimes in the cosmic evolution in which either gravity or antigravity prevails and when going from antigravity to gravity and vice versa a singularity occurs, like in most antigravity contexts [38-41]. It is worth searching theoretical constructions in which such a singularity is avoided. This would probably require some sort of singular conformal transformations between frames, or some singularity of the Lagrangian, a task we hope to address in the near future.

Finally, it is worth discussing the results and also the cosmological implications of our results. The main goal of this paper was to demonstrate all possible cases in which antigravity might appear in modified theories of gravity. As we explicitly demonstrated, in the case of $F(R)$ theories, although the initial Jordan frame $F(R)$ theory had no antigravity (recall the condition $F^{\prime}(R)>0$ which actually guarantees this), 
antigravity might show up when the Jordan frame equivalent theory is considered, modified in the way we explicitly showed in the text. This is one of the new and notable results of this paper. In the case of Brans-Dicke model, introducing by hand a term that causes antigravity, then antigravity might or might not appear in the resulting theory. The latter depends strongly on the value of the antigravity parameter $\mathscr{B}$. In principle, antigravity is a generally unwanted feature in modified theories of gravity and thus it can be considered less harmful if it occurs in the very early universe, prior to inflation. Indeed, this is exactly what happens in all the cases we explicitly demonstrated in the text. However, antigravity is rather difficult to detect experimentally, unless there exists some mechanism of creation of a primordial black hole during the antigravity regime that could retain some information in terms of some sort of gravitational memory [44]. The evaporation of this black hole could reveal the value of the gravitational constant at the time it was created. A well posed question may be to ask how such a compact gravitational object could be created in an antigravity regime. The answer to this could be that antimatter behaves somehow different in antigravity regimes, so it could probably play a prominent role in such a scenario. However, we have to admit that this is just a speculation, since after antigravity occurs, the universe experiences a gravitational regime with a spacetime singularity at the moment of transition. We cannot imagine how a compact gravitational object (if any) could react under such severe conditions.

\section{Conflict of Interests}

The authors declare that there is no conflict of interests regarding the publication of this paper.

\section{References}

[1] S. Nojiri and S. D. Odintsov, "Introduction to modified gravity and gravitational alternative for dark energy," International Journal of Geometric Methods in Modern Physics, vol. 4, no. 1, pp. 115-145, 2007.

[2] A. De Felice and S. J. Tsujikawa, “F(R) theories," Living Reviews in Relativity, vol. 13, p. 3, 2010.

[3] T. P. Sotiriou and V. Faraoni, " $f(R)$ theories of gravity," Reviews of Modern Physics, vol. 82, p. 451, 2010.

[4] S. Nojiri and S. D. Odintsov, "Unified cosmic history in modified gravity: from $F(R)$ theory to Lorentz non-invariant models," Physics Reports, vol. 505, pp. 59-144, 2011.

[5] K. Bamba, S. Capozziello, S. Nojiri, and S. D. Odintsov, "Dark energy cosmology: the equivalent description via different theoretical models and cosmography tests," Astrophysics and Space Science, vol. 342, no. 1, pp. 155-228, 2012.

[6] S. Capozziello and M. de Laurentis, "Extended Theories of Gravity," Physics Reports, vol. 509, no. 4-5, pp. 167-321, 2011.

[7] S. Capozziello, S. Nojiri, S. D. Odintsov, and A. Troisi, "Cosmological viability of $F(R)$-gravity as an ideal fluid and its compatibility with a matter dominated phase," Physics Letters B, vol. 639, no. 3-4, pp. 135-143, 2006.

[8] S. Nojiri and S. D. Odintsov, "Modified gravity with $\ln R$ terms and cosmic acceleration," General Relativity and Gravitation, vol. 36, p. 1765, 2004.
[9] S. Nojiri and S. D. Odintsov, "Modified f(R) gravity consistent with realistic cosmology: from matter dominated epoch to dark energy universe," Physical Review D, vol. 74, Article ID 086005, 2006.

[10] S. Tsujikawa, "Observational signatures of $f(R)$ dark energy models that satisfy cosmological and local gravity constraints," Physical Review D, vol. 77, Article ID 023507, 2008.

[11] S. Nojiri and S. D. Odintsov, "Unifying inflation with $\Lambda$ CDM epoch in modified $F(R)$ gravity consistent with solar system tests," Physics Letters B, vol. 657, pp. 238-245, 2007.

[12] A. A. Starobinsky, "Disappearing cosmological constant in $\mathrm{f}(\mathrm{R})$ gravity," JETP Letters, vol. 86, no. 3, pp. 157-163, 2007.

[13] S. M. Carroll, V. Duvvuri, M. Trodden, and M. S. Turner, "Is cosmic speed-up due to new gravitational physics?" Physical Review D, vol. 70, Article ID 043528, 2004.

[14] O. Bertolami and R. Rosenfeld, "The higgs portal and an unified model for dark energy and dark matter," International Journal of Modern Physics A, vol. 23, no. 30, article 4817, 2008.

[15] A. Capolupo, S. Capozziello, and G. Vitiello, "Dark energy, cosmological constant and neutrino mixing," International Journal of Modern Physics A, vol. 23, no. 31, pp. 4979-4990, 2008.

[16] P. K. S. Dunsby, E. Elizalde, R. Goswami, S. Odintsov, and D. S. Gomez, " $\Lambda$ CDM universe in $f(R)$ gravity," Physical Review $D$, vol. 82, Article ID 023519, 2010.

[17] E. I. Guendelman and A. B. Kaganovich, "Exotic low density fermion states in the two measures field theory: neutrino dark energy," International Journal of Modern Physics A, vol. 21, p. 4373, 2006.

[18] G. Cognola, E. Elizalde, S. Nojiri, S. D. Odintsov, L. Sebastiani, and S. Zerbini, "Class of viable modified $f(R)$ gravities describing inflation and the onset of accelerated expansion," Physical Review D, vol. 77, no. 4, Article ID 046009, 2008.

[19] S. K. Srivastava, "Gravitational origin of phantom dark energy and late cosmic acceleration," International Journal of Modern Physics A, vol. 22, p. 1123, 2007.

[20] S. Capozziello, V. F. Cardone, S. Carloni, and A. Troisi, "Curvature quintessence matched with observational data," International Journal of Modern Physics D, vol. 12, no. 10, pp. 1969-1982, 2003.

[21] S. Capozziello, "Curvature quintessence," International Journal of Modern Physics D, vol. 11, pp. 483-492, 2002.

[22] P. J. E. Peebles and B. Ratra, "The cosmological constant and dark energy," Reviews of Modern Physics, vol. 75, no. 2, pp. 559606, 2003.

[23] V. Faraoni, "Superquintessence," International Journal of Modern Physics D, vol. 11, p. 471, 2002.

[24] E. J. Copeland, M. Sami, and S. Tsujikawa, "Dynamics of dark energy," International Journal of Modern Physics D, vol. 15, no. 11, pp. 1753-1935, 2006.

[25] S. Nojiri and S. D. Odintsov, "Modified gravity with negative and positive powers of curvature: Unification of inflation and cosmic acceleration," Physical Review D, vol. 68, Article ID 123512,2003

[26] J. P. Morais Graca and V. B. Bezerra, "Gravitational field of a rotating global monopole in $f(R)$ theory," Modern Physics Letters A, vol. 27, no. 31, Article ID 1250178, 2012.

[27] M. Sharif and S. Arif, "Static cylindrically symmetric interior solutions in $\mathrm{f}(\mathrm{R})$ gravity," Modern Physics Letters A, vol. 27, no. 25, Article ID 1250138, 12 pages, 2012. 
[28] S. Asgari and R. Saffari, "Vacuum solution of a linear redshift based correction in $\mathrm{f}(\mathrm{R})$ gravity," General Relativity and Gravitation, vol. 44, no. 3, pp. 737-750, 2012.

[29] K. A. Bronnikov, M. V. Skvortsova, and A. A. Starobinsky, "Notes on wormhole existence in scalar-tensor and $F(R)$ gravity," Gravitation and Cosmology, vol. 16, no. 3, pp. 216-222, 2010.

[30] E. V. Arbuzova and A. D. Dolgov, "Explosive phenomena in modified gravity," Physics Letters, Section B, vol. 700, no. 5, pp. 289-293, 2011.

[31] C.-C. Lee, C.-Q. Geng, and L. Yang, "Singularity phenomena in viable $\mathrm{f}(\mathrm{R})$ gravity," Progress of Theoretical Physics, vol. 128, no. 2, pp. 415-427, 2012.

[32] T. Harko, F. S. N. Lobo, S. Nojiri, and S. D. Odintsov, " $F(R, T)$ gravity," Physical Review D, vol. 84, no. 2, Article ID 024020, 2011.

[33] O. Bertolami, C. G. Böehmer, T. Harko, and F. S. N. Lobo, "Extra force in $f(R)$ modified theories of gravity," Physical Review D, vol. 75, Article ID 104016, 2007.

[34] Z. Haghani, T. Harko, F. S. N. Lobo, H. R. Sepangi, and S. Shahidi, "Further matters in space-time geometry: $f\left(R, T, R_{\mu \nu} T^{\mu \nu}\right)$ gravity," Physical Review D, vol. 88, Article ID 044023, 2013.

[35] M. Sharif and M. Zubair, "Study of Bianchi I anisotropic model in $f(R, T)$ gravity," Astrophysics and Space Science, vol. 349, no. 1, pp. 457-465, 2014.

[36] V. Faraoni, Cosmology in Scalar-Tensor Gravity, Kluwer Academic Publishers, Amsterdam, The Netherlands, 2004.

[37] Y. Fujii and K.-I. Maeda, The Scalar-Tensor Theory of Gravitation, Cambridge University Press, Cambridge, UK, 2004.

[38] P. Caputa, S. S. Haque, J. Olson, and B. Underwood, "Cosmology or catastrophe? A non-minimally coupled scalar in an inhomogeneous universe," Classical and Quantum Gravity, vol. 30, no. 19, 2013.

[39] I. Bars, S. H. Chen, P. J. Steinhardt, and N. Turok, "Antigravity and the big crunch/big bang transition," Physics Letters B, vol. 715, no. 1-3, pp. 278-281, 2012.

[40] J. J. M. Carrasco, W. Chemissany, and R. Kallosh, "Journeys through antigravity?" Journal of High Energy Physics, vol. 1401, p. 130, 2014.

[41] K. Bamba, S. Nojiri, S. D. Odintsov, and D. Saez-Gomez, "Possible antigravity regions in $\mathrm{F}(\mathrm{R})$ theory?" Physics Letters $B$, vol. 730, pp. 136-140, 2014.

[42] Y. Fujii, "Choosing a conformal frame in scalar tensor theories of gravity with a cosmological constant," Progress of Theoretical Physics, vol. 99, pp. 599-622, 1998.

[43] M. A. Skugoreva, A. V. Toporensky, and S. Yu, "Global stability analysis for cosmological models with non-minimally coupled scalar fields," http://arxiv.org/abs/1404.6226.

[44] J. D. Barrow, "Gravitational memory?” Physical Review D, vol. 46, no. 8, pp. R3227-R3230, 1992. 

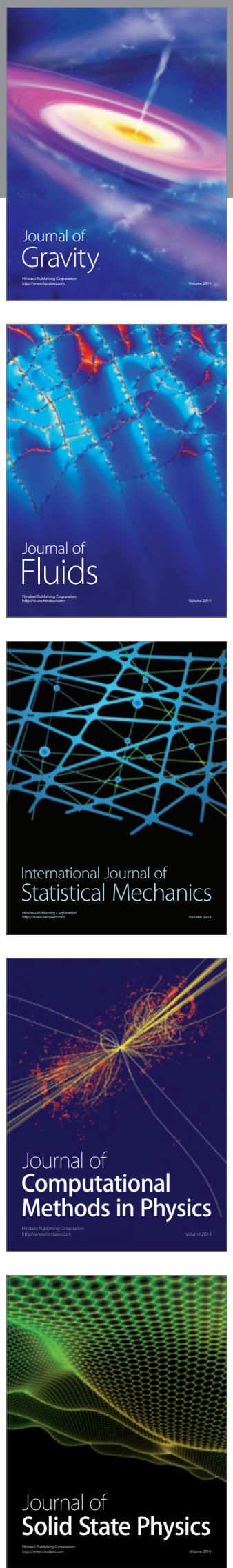

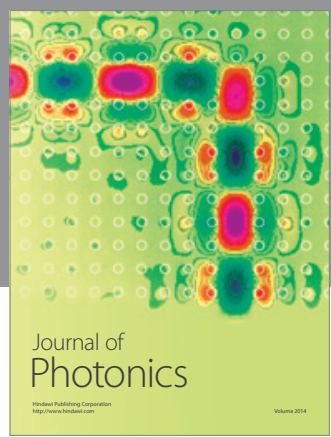

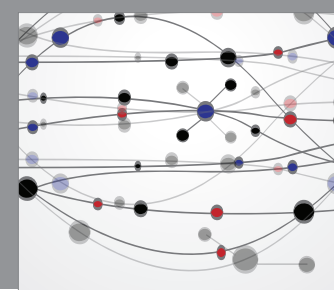

The Scientific World Journal

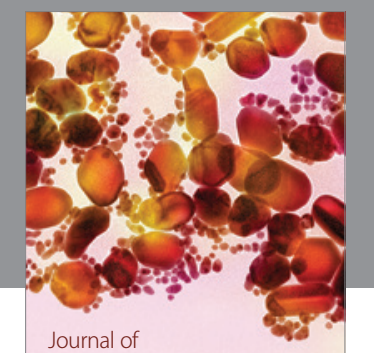

Soft Matter
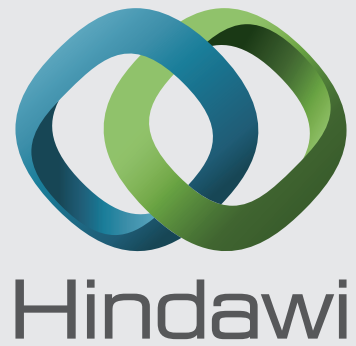

Submit your manuscripts at

http://www.hindawi.com
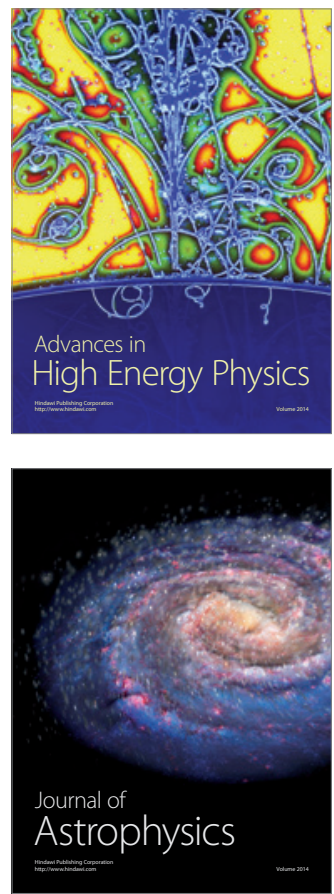
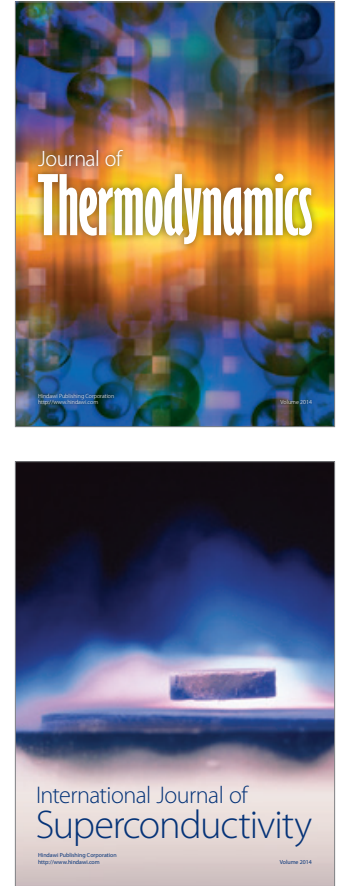
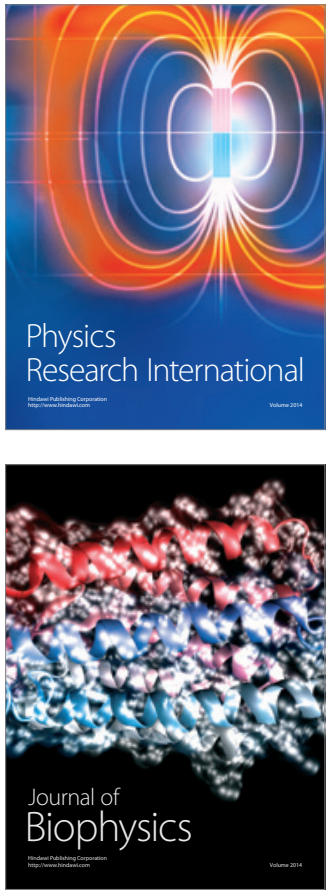
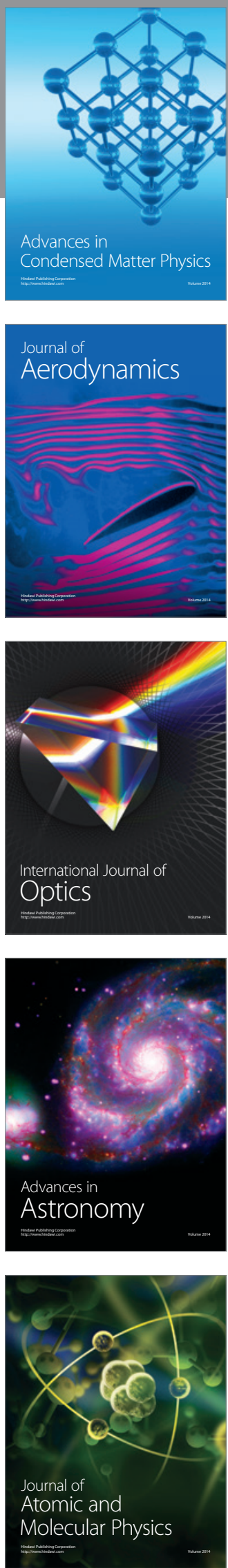